\title{
Protein/Amino Acid Nutrition, Non-Animal
}

National Cancer Institute

\section{Source}

National Cancer Institute. Protein/Amino Acid Nutrition, Non-Animal. NCI Thesaurus.

Code $C 15845$.

Study of dietary non-animal protein/amino acids in relation to cancer. 